\title{
Microsatellite instability in Endometrial carcinoma.
}

\author{
Sharanjit Singh, Prateek Kinra*, Aman Kumar and Onkar Singh Hothi \\ Dept of Pathology, Armed Forces Medical College
}

\begin{abstract}
Background: Endometrial carcinoma is the commonest gynaecological malignancy in the western countries with a standardised incidence of 8 per 100000 women. In India and Southeast Asia, the incidence of endometrial carcinoma is low but it is increasing due to increasing prevalence of obesity, diabetes, early menarche, late menopause, late marriage and declining birth rate. The study aimed to identify the role of microsatellite instability in endometrial carcinoma. Although MSI has been studied extensively in colorectal carcinoma there have been very few studies in southeast asian region regarding association of MSI and prognosis in endometrial carcinoma. To the best of our knowledge this is first study in India.

Method: A Descriptive study in which 40 patients of endometrial carcinoma were studied. MSI was detected using immunohistochemistry (MSH-1, PMS-2, MSH-2, MSH-6). Statistical Analysis was done using SPSS software and fisher exact test was used to calculate $\mathrm{p}$ value. $\mathrm{p}$ value less than. $05 \%$ was considered significant.
\end{abstract}

Results: Overall prevalence of microsatellite instability was $40 \%$. Microsatellite instability was associated with higher tumor grade, myometrial invasion $>50 \%$ and presented in initial stages compared to microsatellite stable tumors.

Conclusion: Our study showed statistically significant association between microsatellite instability and Figo staging, tumor grade and myometrial invasion.

Keywords: Endometrial Carcinoma, Microsatellite Instability, Lynch Syndrome, Colorectal Carcinoma, Carcinogenesis.

\section{Introduction}

Microsatellites are repeat sequences of several DNA bases. They are generally used for paternity testing and other forensic investigations as they are found both in exon and intron regions. As they have repeat structure they are more prone to slippage and thus any error in these regions that take place during replication are repaired by DNA mismatch repair genes (MMR) which are mainly MLH1, MSH2, MSH6, PMS2. ${ }^{[1]}$ In tumors, microsatellite repeat number is different from that in normal tissues which is known as microsatellite instability. MSI has been related to carcinogenicity in various tumors including Lynch syndrome. The role of MSI in colorectal carcinoma has been very well studied with a prevalence of $12-24 \%$ and it is documented that colorectal tumors with MSI behave in a different way clinically when compared to tumors without MSI. ${ }^{[2,3]}$ MSI positivity in colorectal carcinoma is associated with favourable prognosis. Studies of MSI in endometrial carcinoma have been very few. In a study by Caduff et al it was found that endometrial tumors positive for MSI have a high grade and a poor prognosis. ${ }^{[4]}$ On the basis of tumor histology, biology and clinical features endometrial cancers are divided into two major types. Type 1 endometrial cancer is more common (70-80\%), is hormone-sensitive and occurs commonly in women exposed to estrogen. It is generally associated with a higher level of tumor differentiation and has a good prognosis. Type 2 endometrial cancer is less common (20-30\%) and includes serous and clear cell histology. It is characterized by poor level of differentiation and has a higher probability of myometrial invasion with poor prognosis and behaves more aggressively. In addition to these histological subtypes endometrial carcinoma is also classified on basis of molecular alterations one of which is MSI. In 1998 the National Cancer Institute (USA) recommended panel of 05 MSI markers for the determination of MSI. The tumor is called MSI-high if it shows instability in at least 02 markers out of 05; MSI-low if 1 out of 05 and MSI-stable if none. MSI association with prognosis in endometrial carcinoma is not clearly understood. The purpose of our study is to add upon existing knowledge and to compare clinical characteristics and prognosis in endometrial tumors with and without MSI.

\section{Materials and Methods}

Data Collection: 40 patients who were treated for endometrial carcinoma at tertiary care hospital in western Maharashtra between January 2017 and March 2020 were included in the study. The institute ethical clearance was sought before initiation of study. Review blocks were used for study of MSI after permission from head of the institution. The histological type was classified using World Health Organisation criteria and surgical staging 
was determined using the International Federation of Obstetrics and Gynaecology (FIGO).$^{[5]}$

Immunohisochemistry: Immunohistochemical staining was performed on formalin -fixed paraffin embedded specimens using standard avidin-biotin method. Positive controls were sections known to express the investigated antigens, whereas negative controls were obtained by omitting the primary antibodies. Normal staining pattern for MLH1, MSH2, MSH6 and PMS2 is nuclear (Fig15). Evaluable staining was available for all 40 cases. Some cases showed a weak cytoplasmic staining but they were considered negative. To investigate the observer reproducibility all cases were seen by same observer twice and by two different observers.

Statistics: Comparisons of groups was done using fisher exact test. Only cases with conclusive results available for all four IHCs were included. Data was analysed using SPSS software package.

\section{Results}

Of the total 40 endometrial tumors, 34were endometrioid while others 6 included serous papillary (4), high grade serous (1) and clear cell (1). Out of 40 patients $24(60 \%)$ were MS stable whereas $16(40 \%)$ were MS instable (Table 1). Amongst MS instable tumors, 10 out of 16 showed loss of only 2 markers, 1 out of 16 showed loss of single marker, 2 out of 16 showed loss 3 markers and 3 out of 16 showed loss of all 4 markers. Age in our study ranged from 35 to 80 years with mean age of 57 years. $18(45 \%)$ of 40 patients were less than 57 years of age. $37.50 \%$ of MS stable patients were less than 57 years of age compared to $56.25 \%$ of MS instable patients which shows that MS instable tumors tend to occur in younger age group. 03 of $24(12.50 \%)$ MS stable patients were premenopausal compared to 06 of $16(37.50 \%)$ of MS instable patients who were premenopausal.

We also found that 02 of $16(12.50 \%)$ of MS instable tumor presented in advanced stage III-IV disease, whereas 11 out of 24(45.84\%) MS stable patients presented with advanced stage disease. Conversely $87 \%$ of MS instable patients presented in Stage I-II versus 54\% of MSI stable tumors. There was also a trend for higher grade (G2 and G3) in MS instable tumors $(68.75 \%)$ compared to MS stable tumors (25\%). Myometrial invasion greater than 50 percent was seen in $50 \%$ of MS instable tumors compared to $12.50 \%$ of MS stable tumors. Lymphovascular invasion was present in $25 \%$ of MS instable tumors compared to $8.34 \%$ of MS stable tumors. We found out that 04 of 40 patients $(10 \%)$ had family history of carcinoma.

One of our patients who presented with endometrial carcinoma at age of 45 also gave history of carcinoma colon and had all four markers negative. Out of total 06 non endometrioid tumors (Table 2) five were MS stable whereas one was MS instable (Small cell).

\section{Discussion}

Modern medicine has found out molecular basis for carcinogenesis in every tumor and this has led to the discovery of biomarkers for each tumor. ${ }^{[6]}$ Tumorigenesis of colorectal carcinoma has been known to proceed through a series of genetic alterations involving protooncogenes and tumor suppressor genes. ${ }^{[7]}$ One such biomarker involved in colorectal carcinoma is MSI, germline mutations of which have been found in patients with hereditary nonpolyposis colorectal cancer(HNPCC). In the present study, we evaluated the MSI status of endometrial cancers in Indian patients. To the best of our knowledge this is the first study of this type in India. About $20 \%$ endometrial carcinoma have microsatellite instability though only $2-5 \%$ of these are associated with Lynch syndrome. ${ }^{[8,9]}$ Most of MS instable endometrial cancers are endometrioid with occasional other types being MS instable. ${ }^{[10,11]}$ Abnormal expression of MSI in our population was $40 \%$ which is quite high as compared to studies in other parts of the world. A Study by Atif Ali Hashmi et al in Pakistan found MSI instability in $44 \%$ of patients..$^{[12]}$

In colorectal carcinoma, MSI is associated with older age, female sex, and other clinicopathological parameters such as prevalence in the proximal colon, mucinous differentiation, lymphocytic infiltration and low pathological stage. ${ }^{[13,14]}$ In endometrial carcinoma most of research has concluded that MS instable phenotype has been associated with Type 1 or endometrioid type, high tumor grade and, greater myometrial invasion. ${ }^{[15,16]}$ Our study too showed a greater percentage of MS instable patients with higher tumor grade as compared to MS stable patients. Our study also showed that majority of MS instable tumors present in lower FIGO stage compared to MS stable which present at a late stage. As per our study MS instable tumors have a higher prevalence in premenopausal compared to MS stable tumors in pre-menopausal patients.

The MMR protein occurs in two complexes MLH1/ PMS2 and MSH2/MSH6. MLH1 and MSH2 are stable without their counterparts but PMS2 and MSH6 require their counterparts for stability. ${ }^{[17]}$ Thus PMS2 and MSH6 are not expressed if their counterparts are not expressed, hence causing loss of MLH1/PMS2 in case of MLH1 defect and loss of MSH2/MSH6 in case of MSH2 defect. With the loss of MSH6 only or PMS2 only germline testing for each is carried. ${ }^{[18,19]}$ In case MSH2 is negative further testing for germline mutations in EPCAM is indicated. ${ }^{[20]}$ In case of loss of MLH1 on IHC, it can be 
Table 1: Association of various clinicopathological factors in endometrial tumors with and without microsatellite instability.

\begin{tabular}{|c|c|c|c|c|c|c|c|}
\hline Category & No of Patients & $\%$ of Total & MS Stable & $\%$ & $\begin{array}{l}\text { MSI } \\
\text { Instable }\end{array}$ & $\%$ & $p$ value \\
\hline & 40 & 100 & 24 & 60.00 & 16 & 40.00 & \\
\hline \multicolumn{8}{|l|}{ Age } \\
\hline$<57$ & 18 & 45.00 & 09 & 37.50 & 09 & 56.25 & \multirow[t]{2}{*}{0.334} \\
\hline$>57$ & 22 & 55.00 & 15 & 62.50 & 07 & 43.75 & \\
\hline \multicolumn{8}{|l|}{ Menopause } \\
\hline Pre & 09 & 22.50 & 03 & 12.50 & 06 & 37.50 & \multirow[t]{2}{*}{0.119} \\
\hline Post & 31 & 77.50 & 21 & 87.50 & 10 & 62.50 & \\
\hline \multicolumn{8}{|l|}{ Figo Stage } \\
\hline $1+I I$ & 27 & 70.00 & 13 & 54.16 & 14 & 87.50 & \multirow[t]{2}{*}{0.040} \\
\hline III+IV & 13 & 30.00 & 11 & 45.84 & 02 & 12.50 & \\
\hline \multicolumn{8}{|l|}{ Tumor grade } \\
\hline G1 & 23 & 57.50 & 18 & 75.00 & 05 & 31.25 & \multirow[t]{2}{*}{0.009} \\
\hline $\mathrm{G} 2+\mathrm{G} 3$ & 17 & 42.50 & 06 & 25.00 & 11 & 68.75 & \\
\hline \multicolumn{8}{|c|}{ Myometrial Invasion } \\
\hline$<50 \%$ & 29 & 72.50 & 21 & 87.50 & 08 & 50.00 & \multirow[t]{2}{*}{0.013} \\
\hline$>50 \%$ & 11 & 27.50 & 03 & 12.50 & 08 & 50.00 & \\
\hline \multicolumn{8}{|c|}{ Lymphovascular Invasion } \\
\hline Absent & 34 & 85.00 & 22 & 91.66 & 12 & 75.00 & \multirow[t]{2}{*}{0.195} \\
\hline Present & 06 & 15.00 & 02 & 08.34 & 04 & 25.00 & \\
\hline \multicolumn{8}{|c|}{ Lymph Node Metastasis } \\
\hline Absent & 37 & 95.00 & 23 & 95.83 & 14 & 87.50 & \multirow[t]{2}{*}{0.553} \\
\hline Present & 03 & 05.00 & 01 & 04.17 & 02 & 12.50 & \\
\hline \multicolumn{8}{|l|}{ Family History } \\
\hline Absent & 36 & 90.00 & 21 & 87.50 & 15 & 93.75 & \multirow[t]{2}{*}{0.637} \\
\hline Present & 04 & 10.00 & 03 & 12.50 & 01 & 06.25 & \\
\hline \multicolumn{8}{|c|}{ Tumor Histology } \\
\hline Endometrioid & 34 & 87.50 & 19 & 79.16 & 15 & 93.75 & \multirow[t]{2}{*}{0.372} \\
\hline Others & 06 & 12.50 & 05 & 20.84 & 01 & 06.25 & \\
\hline
\end{tabular}

Fisher Exact Test used to calculate p value

Table 2: Pattern of expression of MSI Markers in endometroid and non-endometroid endometrial tumors.

\begin{tabular}{|l|l|l|}
\hline Total No of cases & $\begin{array}{l}\text { Endometrioid } \\
(\mathbf{n = 3 4 )}\end{array}$ & $\begin{array}{l}\text { Others } \\
(\mathbf{n = 0 6})\end{array}$ \\
\hline All markers retained & 19 & 05 \\
\hline Isolated loss of PMS2 & 01 & 00 \\
\hline Loss of MLH1, PMS2 & 10 & 00 \\
\hline Loss of 3 Markers (MLH1, PMS2, MSH6) & 02 & 00 \\
\hline Loss of all 4 markers & 02 & 01 \\
\hline Loss of MSH2, MSH6 & 00 & 00 \\
\hline
\end{tabular}




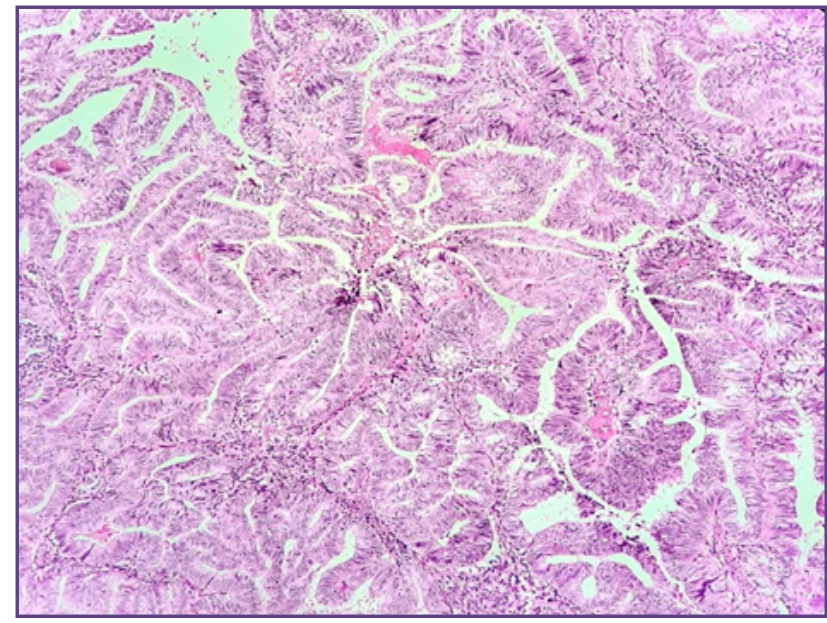

Fig. 1: (a) Well differentiated endometrioid adenocarcinoma. (H\&E 200X).

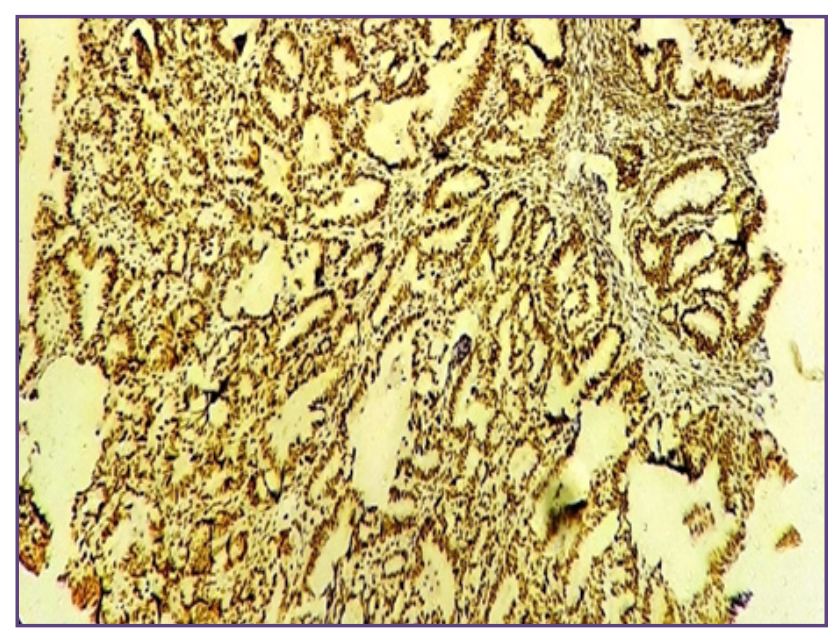

Fig. 3: Tumor cells with retained positivity for PMS2. (200x, DAB used as chromogen).

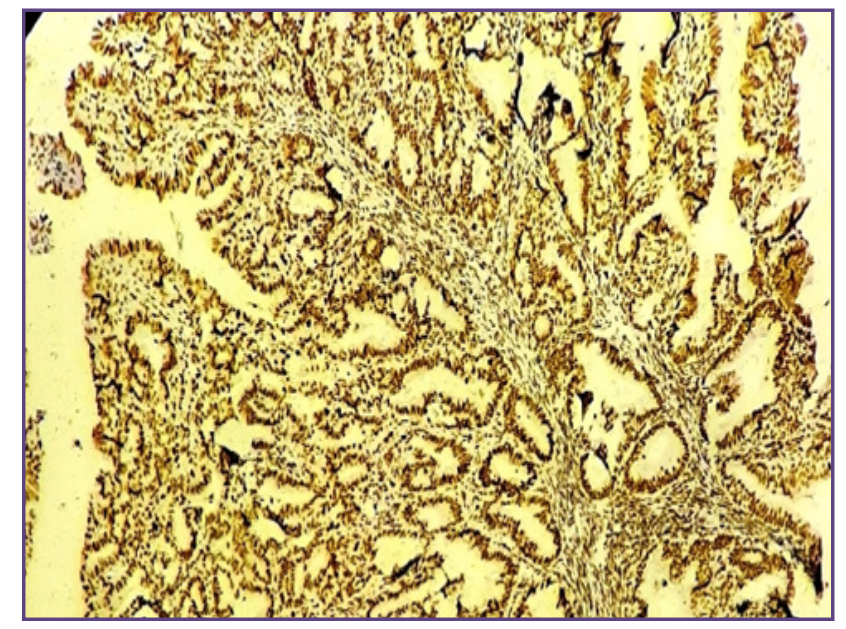

Fig. 2: Tumor cells with retained positivity for MLH1. (200x, DAB used as chromogen).

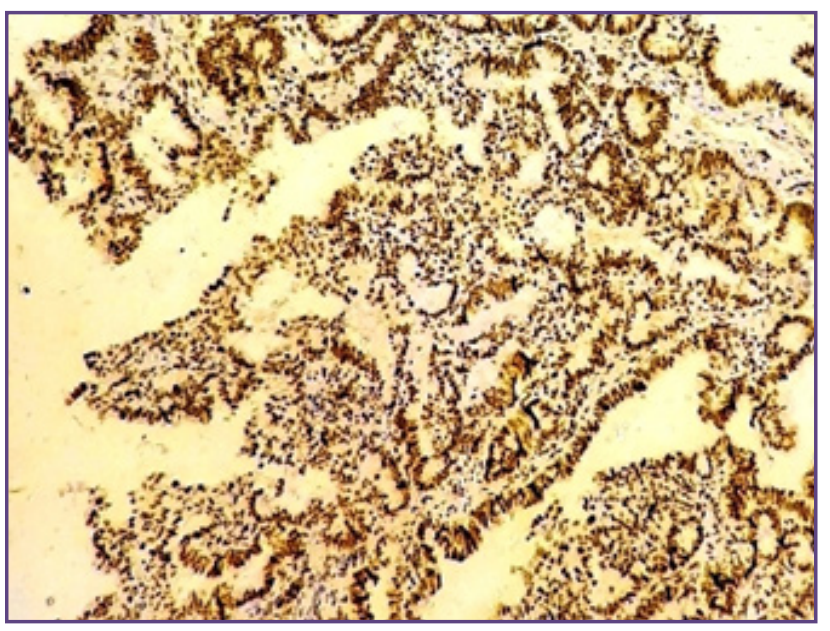

Fig. 4: Tumor cells with retained positivity for MSH2. (200x, DAB used as chromogen).

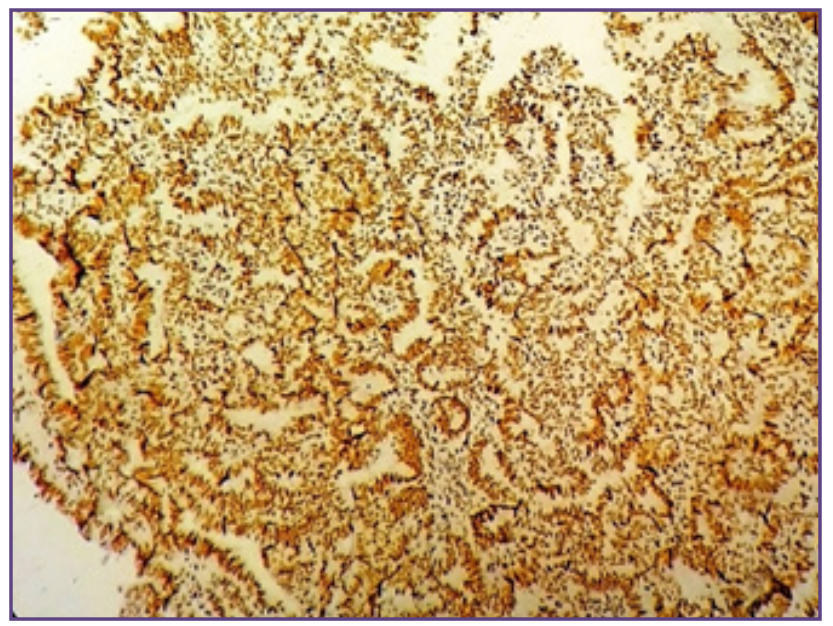

Fig. 5: Tumor cells with retained positivity for MSH6. (200x, DAB used as chromogen). 
due to MLH1 hypermethylation likely sporadic and does not require further testing for Lynch syndrome. ${ }^{[18,19]}$ In such cases, MLH1 hypermethylation is checked and if present it indicates a sporadic cause for MMR defect. MLH1 hypermethylation in endometrial carcinoma is not associated with BRAF and hence BRAF cannot be used as an indicator for sporadic MMR defect to rule out Lynch syndrome. ${ }^{[19]}$ If MLH1 hypermethylation is absent further germline testing for MLH1 is indicated. In cases with strong suspicion for Lynch syndrome with MLH1 hypermethylation germline methylation or germline epimutation is likely. ${ }^{[20]}$ Our study had loss of MLH1/ PMS2 pattern highest among MS instable tumors.

Another significance of MSI testing is the benefit of PDL1 antagonists. MSI-high tumors generally harbour high neoantigen loads, increased immune checkpoint expression such as programmed cell death protein (PD-1), and programmed cell death ligand-1(PDL-1) and an increased number of tumor-infiltrating lymphocytes. Thus, these tumors are good candidates for immunotherapy. It has been seen that MS instable tumors have higher response rates to anti PDL1 therapy compared to MS stable tumors. ${ }^{[21]}$

However, the main limitation of our study was the small sample size. Thus, it is necessary to continue the investigation including a greater number of patients with MS instable tumors.

\section{Conclusion}

Although MSI has been associated with favourable prognosis in colorectal carcinoma patients, the MSI overall impact in endometrial carcinoma is still controversial. According to our study MS instable phenotype is more common in tumors with higher tumor grade, tumors with deep myometrial invasion and those which present in early stage of disease; thus, it may be associated with worse prognosis.

\section{Acknowledgement}

Nil

\section{Funding sources}

Nil

\section{Competing Interests}

Nil.

\section{References}

1. Kunitomi H, Banno K, Yanokura M, Takeda T, Iijima M, Nakamura K, Iida M, Adachi M, Watanabe K, Matoba Y, Kobayashi Y, Tominaga E, Aoki D. New use of microsatellite instability analysis in endometrial cancer. Oncol Lett. 2017;14(3):3297 $\square 3301$.
2. Jernvall P, Makinen MJ, Karttunen TJ, Makela J, Vihko P. Microsatellite instability: impact on cancer progression in proximal and distal colorectal cancers. Eur J Cancer 1999; 35: 197- 201.

3. Gryfe R, Kim H, Hsieh ET, Aronson MD, Holowaty EJ, Bull $\mathrm{SB}$, et al. Tumor microsatellite instability and clinical outcome in young patients with colorectal cancer. N Engl J Med 2000; 342: 69- 77.

4. Caduff RF, Johnston CM, Svoboda $\square$ Newman SM, Poy EL, Merajver SD, Frank TS. Clinical and pathological significance of microsatellite instability in sporadic endometrial carcinoma. Am J Pathol 1996; 148: 1671-8.

5. American Joint Committee on Cancer. Cervix Uteri. In: AJCC cancer staging manual. 8th ed. New York, NY: Springer, 2017; 649-659.

6. Salvesen HB, Haldorsen IS, Trovik J. Markers for individualised therapy in endometrial carcinoma. Lancet Oncol. 2012;13:e353-e361.

7. Kinzler KW, Vogelstein B. Lessons from hereditary colorectal cancer. Cell 1996; 87: 159- 70

8. Meyer LA, Broaddus RR, Lu KH. Endometrial Cancer and Lynch Syndrome: Clinical and Pathologic Considerations. Cancer Control. 2009; 16: 14-22.

9. Rabban JT, Calkins SM, Karnezis AN, Grenert JP, Blanco A, et al. Association of Tumor Morphology With MismatchRepair Protein Status in Older Endometrial Cancer Patients: Implications for Universal Versus Selective Screening Strategies for Lynch Syndrome. Am J Surg Pathol .2014;38: 793-800.

10. Mills AM, Liou S, Ford JM, Berek JS, Pai RK, et al. Lynch Syndrome Screening Should be Considered for all Patients With Newly Diagnosed Endometrial Cancer. Am J Surg Pathol. 2014; 38: 1501-1509.

11. Broaddus RR, Lynch HT, Chen Lm, Daniels MS, Conrad $\mathrm{P}$, et al. Pathologic Features of Endometrial Carcinoma Associated with HNPCC. Cancer. 2006;106: 87-94.

12. Hashmi AA, Mudassir G, Hashmi RN, et al. Microsatellite Instability in Endometrial Carcinoma by Immunohistochemistry, Association with Clinical and Histopathologic Parameters. Asian Pac J Cancer Prev. 2019;20(9):2601 $\square 2606$.

13. Boland CR, Goel A. Microsatellite instability in colorectal cancer. Gastroenterology. 2010;138:2073-87 e3.

14. Hong SP, Min BS, Kim TI, et al. The differential impact of microsatellite instability as a marker of prognosis and tumour response between colon cancer and rectal cancer. Eur J Cancer. 2012;48(8):1235 $\square 1243$

15. Hirasawa A, Aoki D, Inoue J, Imoto I, Susumu N, Sugano $\mathrm{K}$, et al. Unfavorable prognostic factors associated with high frequency of microsatellite instability and comparative genomic hybridization analysis in endometrial cancer. Clin Cancer Res 2003;9:5675-82. 
16. H.J. An, K.I. Kim, J.Y. Kim, J.Y. Shim, H. Kang, T.H. Kim, et al.Microsatellite instability in endometrioid type endometrial adenocarcinoma is associated with poor prognostic indicators. Am J SurgPathol, 31 (2007), pp. $846-853$

17. Vilar E, Gruber SB. Microsatellite Instability in Colorectal Cancer-the Stable Evidence. Nat Rev Clin Oncol. 2010; 7: 153-162.

18. Buchanan DD, Tan YY, Walsh MD. Tumor Mismatch Repair Immunohistochemistry and DNA MLH1 Methylation Testing of Patients With Endometrial Cancer Diagnosed at Age Younger Than 60 Years Optimizes Triage for PopulationLevel Germline Mismatch Repair Gene Mutation Testing. J Clin Oncol. 2014; 32: 90-100.
19. Goodfellow PJ, Billingsley CC, Lankes HA, Ali S, Cohn DE, et al. Combined Microsatellite Instability, MLH1 Methylation Analysis, and Immunohistochemistry for Lynch Syndrome Screening in Endometrial Cancers From GOG210: An NRG Oncology and Gynecologic Oncology Group Study. J Clin Oncol . 2015; 33: 4301-4308.

20. Patil PA . Microsatellite Instability Testing In Endometrial Cancer - A Short Review. J Oncol Res Treat. 2018; 3: 126.

21. Howitt BE, Shukla SA, Sholl LM, et al. Association of polymerase e-mutated and microsatellite-instable endometrial cancers with neoantigen load, number of tumorinfiltrating lymphocytes, and expression of PD-1 and PDL1. JAMA Oncol. 2015;1:1319-23.

*Corresponding author:

Gp Capt (Dr) Prateek Kinra, Professor and Offg HOD, Dept of Pathology, Armed Forces Medical College, Sholapur Road, Pune: 411060, Maharastra Phone: +919945277110

Email: pkinra_in@yahoo.com 